\title{
Sperm extraction and culture techniques bring new hope for men once considered infertile
}

S permatogenesis is a complex process, taking more than a month to create functional sperm in vivo. Men who receive chemotherapy for cancer during childhood often experience disrupted spermatogenesis later in life. In the past, these men have been considered unable to father children. However, new research shows that not only can functional sperm be extracted from the testes of men previously thought infertile, but also that in vitro organ culture techniques can produce functional sperm that yield viable, live offspring in mice.

In the Journal of Clinical Oncology, a group based at the Weill Cornell Medical College describe the microsurgical extraction of sperm from 73 men who had previously received chemotherapy for childhood cancers. The group used testicular sperm extraction (TESE), microdissecting the testis until sperm were found within it. "It was thought possible that some of these men who had 'sterilizing' chemotherapy would have sperm being made within the testis that could be found with a detailed microsurgical search," explains Peter Schlegel, who led the team.

The initial TESE procedure recovered sperm from $37 \%$ of men, with an overall success rate of $43 \%$. The recovered gametes were then used for assistedfertilization using intracytoplasmic sperm injection (ICSI), resulting in a 50\% clinical pregnancy rate and a $42 \%$ live birth rate.

Interestingly, sperm recovery was affected by both the indication for chemotherapy and the medications used for treatment, with a much lower rate of retrieval in men who had been exposed to alkylating agents. The lowest retrieval rate $(14 \%)$ was seen in men who had been treated for sarcoma, whereas men who had undergone testicular cancer therapy had an $85 \%$ retrieval rate, possibly reflecting the less gonadotoxic chemotherapy regimens used to treat the latter.

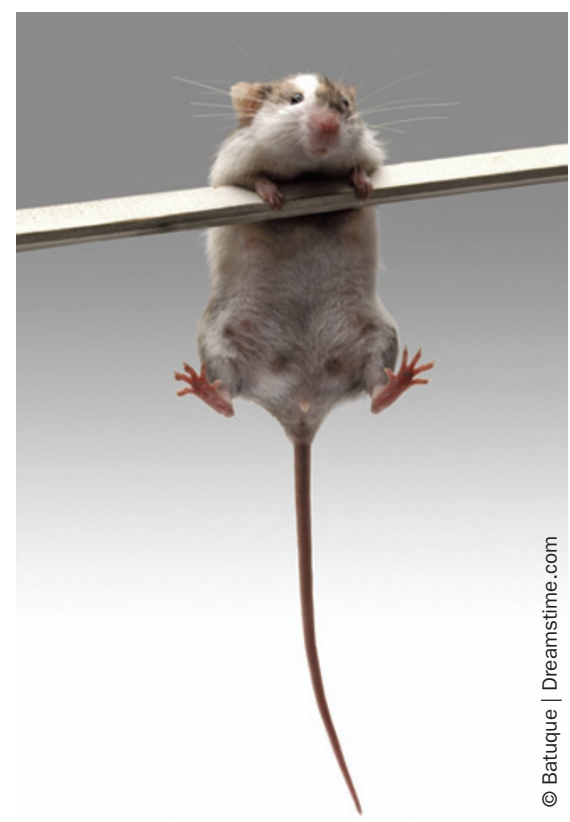

This study is the largest to date that demonstrates TESE and ICSI can be used to treat male infertility associated with childhood chemotherapy, producing viable offspring that are lacking in detectable birth defects.

Further to these clinical developments, a recent article in Nature has described the in vitro creation of functional sperm using organ culture techniques. The researchers responsible, from Yokohama City University Graduate Medical School, are the first to reproduce the maturation and growth of mammalian sperm outside the body, using neonatal mouse testes containing only gonocytes or primitive spermatogonial germ cells.

Using a standard gas-liquid interphase method, the group sought to culture testis tissue fragments from transgenic mice expressing GFP, which would enable them to monitor the progress of spermatogenesis in vitro. Takehiko Ogawa, who led the group, is quick to point out that he was unsure if their groundbreaking experiments would be successful.
"My [initial] optimism changed into pessimism when I thought that the organ culture gas-liquid interphase method may not be able to sustain spermatogenesis because it lacks vascular and lymphatic circulatory systems," he comments.

Despite his concerns, round spermatids (haploid cells) were observed in the cultured testes in initial studies of the organ culture technique. However, the lack of elongating spermatids and sperm cells encouraged the team to modify the culture media to optimize the environment for promoting spermatogenesis. Concerned that the addition of fetal bovine serum to the medium was hindering the process, they began to use KSR as serum replacement. This alteration resulted in the formation of flagellated sperm in the cultured tissues. Furthermore, using these cultured sperm for microinsemination resulted in live births, and these mice were fertile and able to produce viable offspring. The testis fragments could also be preserved in liquid nitrogen and resume spermatogenesis when thawed and cultured.

"My concern disappeared and changed into confidence that gas-liquid interphase methods can support spermatogenesis of other animals, including humans, if the culture media was optimized for them," says Ogawa.

Only time will tell if this method could work for human sperm. However, Ogawa's enthusiasm will surely be mirrored by patients who could benefit from both of these exciting new techniques.

Annette Fenner

Original articles Hsaio, W. et al. Successful treatment of postchemotherapy azoospermia with microsurgical testicular sperm extraction: the Weill Cornell experience. J. Clin. Oncol. doi:10.1200/JC0.2010.33.7808 | Sato, T. et al. In vitro production of functional sperm in cultured mouse testes. Nature 471, 504-507 (2011) 